\title{
COMPARACIÓN DE CUATRO MÉTODOS DE EVALUACIÓN VISUAL DEL RIESGO DE ÁRBOLES URBANOS
}

\section{Comparison of four methods of visual risk tree assessment in urban areas}

\author{
Jorge Reyes de la Barra', Mauricio Ponce-Donoso², Óscar Vallejos-Barra33, \\ Gustavo Daniluk-Mosquera ${ }^{4}$ y Ana Paula Coelho Duarte ${ }^{5}$
}

Reyes de la Barra, J., Ponce-Donoso, M., Vallejo-Barra, O., Daniluk-Mosquera, G. y Coelho-Duarte, A.P. (2018). Comparación de cuatro métodos de evaluación visual del riesgo de árboles urbanos. Colombia Forestal, 21(2), 161-173

Recepción: 19 de octubre de 2017

\section{Resumen}

Cuatro métodos de evaluación visual del riesgo se aplicaron en 30 árboles urbanos con niveles de riesgo en la ciudad de Talca (Chile). Los métodos utilizados fueron: "Avaliação de árvores de risco na arborização de vias públicas de Nova Olímpia" (método Sampaio), "Internation Society of Arboriculture (ISA) Matheny y Clark" (método ISA[A]), "Best Management Practice BMP/ISA" (método ISA[B]) y "Urban tree risk management USDA Forest Service" (método USF). Los resultados fueron estandarizados para su análisis estadístico, se encontraron diferencias significativas y variaciones en los métodos al aplicar el test no paramétrico de Kruskal-Wallis y de Duncan. Se conformaron tres grupos: los métodos Sampaio y USF, que presentaron evaluaciones con riesgo muy alto y alto, respectivamente; el segundo, conformado por USF e ISA(A), presentó evaluaciones de alto a medio riesgo; mientras que un tercer grupo lo conformó ISA(B), con evaluaciones de riesgo bajo. Ya que la ciudad de Talca no cuenta con una metodología propia, se sugiere usar en el área mediterránea de Chile el método ISA(A) o USF, ya que fueron los métodos que presentaron una mejor adecuación a las condiciones locales.
Aprobación: 22 de marzo de 2018

Palabras clave: arbolado urbano, arboricultura, riesgo del árbol urbano, silvicultura urbana.

\begin{abstract}
Four methods of visual risk tree assessment were applied in 30 urban trees with different risk levels, in Talca city (Chile). The methods used were: "Avaliação de árvores de risco na arborização de vias públicas de Nova Olímpia" (Sampaio Method), "International Society of Arboriculture (ISA) Matheny \& Clark" (Method ISA[A]), "Best Management Practice BMP/ISA" (Method ISA[B]) and "Urban tree risk management USDA Forest Service" (Method USF). The results were standardized for statistical analysis, finding significant differences and variations between the methods, after applying the nonparametric Kruskal-Wallis and Duncan test. Three groups were formed; the Sampaio and USF methods, which presented very high and high risk assessments, respectively; the second one was conformed by USF and ISA (A), which presented high to medium risk assessments; while the third group was shaped by ISA (B) with low risk assessments. Since the Talca city does not have its own methodology, it is suggested that the ISA (A) or USF method could be used in
\end{abstract}

\footnotetext{
Facultad de Ciencias Forestales, Universidad de Talca. Talca, Chile. jorreyes@utalca.cl

Facultad de Ciencias Forestales, Universidad de Talca. Talca, Chile. mponce@utalca.cl. Autor para correspondencia

Facultad de Ciencias Forestales, Universidad de Talca. Talca, Chile. ovallejo@utalca.cl

Departamento Forestal, Universidad de la República. Montevideo, Uruguay. gdaniluk@fagro.edu.uy

Departamento Forestal, Universidad de la República. Montevideo, Uruguay. paula.coelho@fagro.edu.uy
} 
the Mediterranean area of Chile, because they were the methods presenting the best adaptation to local conditions.
Key words: urban trees, arboriculture, urban tree risk, urban forestry.

\section{INTRODUCCIÓN}

El concepto de silvicultura urbana se origina en los años sesenta en Norteamérica y en Europa en los años ochenta (Pauleit et al., 2002; Konijnendijk, Kjell, Randrup y Schipperijn, 2005). Se circunscribe a aquellas áreas arboladas o árboles que se ubican en sectores urbanos y periurbanos (Tyrväinen, Silvennoinen y Kolehmainen, 2003) e incluye plantación, cuidado y manejo de los árboles en las ciudades para asegurar múltiples beneficios para los habitantes urbanos (Miller, Hauer y Werner, 2015; FAO, 2017). La presencia del arbolado urbano tiene un valor tanto intrínseco como monetario (Ponce-Donoso y Vallejos-Barra, 2016) lo que les otorga un valor patrimonial a las ciudades. Su gestión es posible bajo una aproximación integral, que involucra planificación, diseño, desarrollo, conservación y manejo; realizado conjuntamente tanto por entidades públicas y privadas, como por los propios habitantes (Forrest, 2002; Pauleit et al., 2002). Estos deben inscribirse según los principios de la biología arbórea, que garantice a largo plazo y bajo óptimas condiciones su supervivencia, haciendo uso del análisis urbanístico y del planeamiento como instrumento multidisciplinario para afrontar la proyección de la ciudad (Bonells, 2003).

Los árboles urbanos conllevan un riesgo o potencial de fallo que puede poner a las personas en peligro, dañar sus propiedades o ambos, aspecto que ha ido creciendo tanto en las personas como en los administradores municipales (Koeser, Hauer, Miesbauer y Peterson, 2016). Las situaciones de riesgo del árbol se pueden controlar parcialmente, pero no se puede eliminar completamente sin suprimir cada árbol. Por ello, el desafío está en desarrollar una adecuada gestión con el fin de asegurar un nivel de riesgo aceptable (Tomao et al., 2015) para sus gestores, compañías de seguros y la sociedad en general. Por ejemplo, un árbol longevo sin mantenimiento tiene más probabilidad de fallar (O'Brien, 2003) por lo que es importante identificar dónde un árbol se ha convertido en un riesgo inaceptable a consecuencia de su deterioro natural, por estrés hídrico o térmico, contaminación atmosférica, urbanización, ataque de plagas o enfermedades (Restrepo, Moreno y Hoyos, 2015) o producto de las inadecuadas intervenciones $u$ otros elementos que concomitan en su riesgo.

La eliminación total del riesgo del arbolado urbano es una actividad económica y técnicamente inviable; por lo cual el gestor debe hacer uso de herramientas eficientes que orienten sus intervenciones, como es el caso de las metodologías de evaluación del riesgo de árboles urbanos; entre ellos, los métodos descritos en Mattheck y Breloer (1994), Matheny y Clark (1994), Pokorny (2003) y Ellison (2005). Actualmente, estatutos y ordenanzas municipales obligan a un mayor nivel de formación para gestionar los árboles en la ciudad (Van Wassenaer y Richardson, 2009).

La National Tree Safety Group (NTSG, 2011) define peligro como una situación o condición con el potencial de causar daño, dando a entender que cualquier parte del árbol, tronco, ramas o copa puede fallar estructuralmente, colapsar y caer sobre una persona o propiedad, causando lesiones o daños. Por otro lado, Calaza e Iglesias (2016) definen riesgo como contingencia o proximidad de un daño, mientras que peligro es un riesgo o contingencia inminente de que suceda algún mal. Por tanto, un árbol peligroso es un árbol con riesgo inminente de que suceda algún mal, es decir, con proximidad inminente de producir un daño. De tal 
manera que el riesgo puede clasificarse en varios niveles, por ejemplo, como en la guía BMP/ISA (Smiley, Matheny y Lilly, 2011), que clasifica en bajo, moderado, alto y extremo.

Una zona de riesgo alto incluye áreas con un concurrido grado de uso, ya sea debido a la afluencia de personas, automóviles y estructuras permanentes, denominadas dianas, correspondiendo a zonas prioritarias para inspeccionar (Ellison, 2005). Las zonas de riesgo medio tienen uso intermitente, cuya prioridad de inspección se basa en la cantidad y el tipo de uso. La zona de riesgo bajo carece de vehículos o estructuras y un bajo uso de visitantes, por lo que tienen una baja prioridad de inspección (Angwin, Cluck, Zambino, Oblinger y Woodruff, 2012). Lo anterior se tiene como causa que lograr cero riesgos se vuelve costoso en el tiempo, conlleva extracciones prematuras de árboles, reemplazos más frecuentes y la pérdida de beneficios que los árboles maduros proporcionan (Hauer y Johnson, 2003; Geiger, King y Hartel, 2004).

No todos los árboles defectuosos pueden ser detectados, corregidos o eliminados; aunque se puede reconocer fácilmente la mayoría de los defectos y síntomas. Sin embargo, hay problemas en raíces y algunos defectos internos que no son perceptibles y pueden requerir inspecciones en profundidad, además del uso de herramientas de diagnóstico especializadas (Pokorny, 2003; Norris, 2007) como tomógrafo, resistógrafo y georradar, y los sensores para análisis estructural dinámico de los árboles bajo carga de vientos (James, 2010), pero a costos considerables. Cabe señalar que muchos defectos pueden ser evaluados mediante una inspección visual, identificando y comprendiendo los indicadores de peligrosidad potencial (Calaza e Iglesias, 2016); los defectos son señales visibles de un árbol que tiene el potencial de fallar de manera predecible pues los defectos muestran dónde es probable que falle el árbol. Así, se identifican siete categorías de defectos potenciales de fallo: madera descompuesta, grietas, problemas en las raíces, uniones débiles de ramas, cancros, arquitectura pobre del árbol y ramas, copa o árboles muertos (Pokorny, 2003; Calaza e Iglesias, 2016).

En la actualidad es posible encontrar diversos estudios en los que se evalúa el desempeño de diferentes métodos, visuales, cuantitativos como cuali-cuantitativos, en los cuales participan arboristas acreditados y con experiencia, cuyos resultados muestran una amplia variabilidad (Norris, 2007; Koeser y Smiley, 2017). Por su parte, Koeser, Hauer, Klein y Miesbauer (2017) evalúan la probabilidad de falla en el arbolado urbano haciendo uso de diferentes técnicas, en los niveles básico, intermedio y avanzado, apoyados en la Norma A300 (ANSI, 2011) y en la guía de buenas prácticas para la gestión del riesgo BMP de la ISA (Smiley, Matheny, N. y Lilly, 2011); encontraron que el básico y avanzado tienen menos diferencias entre sí, comparativamente con el intermedio, destacando que ningún nivel de evaluación redujo la variabilidad de los evaluadores participantes. Norris (2007) identifica 23 métodos de evaluación del riesgo en árboles urbanos, varios de ellos de tipo privado, y evalúa 15 en un estudio desarrollado en Melbourne (Australia), con la finalidad de identificar la influencia individual en los criterios de evaluación, encontrándose diferencias debido a su estructura, peso relativo de las variables y tipos de categorizaciones. En el estudio, los métodos de Matheny y Clark (1994) y de Pokorny (2003) presentan desviaciones estándar bajas ( $20 \%$ y $22 \%$ respectivamente), ocupando el segundo y tercer lugar, cuya correlación entre métodos es de 0.63 .

En el contexto descrito, es necesario analizar métodos de evaluación para contribuir en la selección más practicable en contextos locales, a la espera de la adaptación de un método ya existente o bien el desarrollo de uno específico. Por ello, este artículo tiene como finalidad comparar cuatro metodologías de evaluación visual del riesgo en arbolado urbano disponibles y factibles de aplicar, ejecutado por un único evaluador, y analizar estadísticamente sus resultados con el fin de proponer cuál de ellos resulta más conveniente. 


\section{MATERIALES Y MÉTODOS}

Se evaluaron árboles de la ciudad de Talca, Región del Maule, Chile; la cual se encuentra a una altitud de $102 \mathrm{~m}$, latitud $35^{\circ} 25^{\prime} 59^{\prime \prime}$ sur y longitud $71^{\circ} 40^{\prime}$ $00^{\prime \prime}$ oeste, cubre una superficie de $51 \mathrm{~km}^{2}$ y posee una población urbana cercana a los 220 mil habitantes (Biblioteca del Congreso Nacional [BCN], s.f.).
Se seleccionaron visualmente 30 árboles en sectores residenciales y comerciales de la ciudad, los cuales aparentemente mostraban diferentes condiciones de riesgo. Los criterios de selección se basaron en parámetros e indicadores de riesgo considerados en los métodos de evaluación elegidos que se encuentran definidos en la tabla 1.

Tabla 1. Información de las especies seleccionadas

\begin{tabular}{|c|c|c|c|}
\hline N. ${ }^{\circ}$ & Especie & Ubicación & Criterio de selección \\
\hline 1 & Morus nigra & 14 1/2 sur con Pasaje 2 poniente & Arquitectura pobre, ramas muertas y grietas \\
\hline 2 & Schinus sp. & 14 sur con 3 poniente & Arquitectura pobre y grietas \\
\hline 3 & Schinus sp. & 15 sur con 3 poniente & Arquitectura pobre \\
\hline 4 & Eucalyptus sp. & $\begin{array}{l}\text { Av. Ignacio Carrera Pinto con Pasa- } \\
\text { je Los Caquis }\end{array}$ & Arquitectura pobre \\
\hline 5 & Schinus sp. & 3 sur con 11 poniente & $\begin{array}{l}\text { Arquitectura pobre, inclinación y carga en ramas indivi- } \\
\text { duales }\end{array}$ \\
\hline 6 & Schinus sp. & 13 sur con 11 poniente & Arquitectura pobre y unión débil de ramas \\
\hline 7 & Acer negundo & 5 sur con 2 oriente & Madera descompuesta y cavidad \\
\hline 8 & Platanus orientalis & 6 sur con 4 oriente & Arquitectura pobre \\
\hline 9 & Platanus orientalis & El Arenal con 1 oriente & Cancro \\
\hline 10 & Acer negundo & 5 sur con 4 oriente & Cavidad, cancro, inclinación y grieta \\
\hline 11 & Acer negundo & 6 sur con 4 oriente & Arquitectura pobre y cancro \\
\hline 12 & Acer negundo & 1 norte con 4 oriente & Arquitectura pobre, cancro y muerte regresiva de copa \\
\hline 13 & Acer negundo & 2 norte con 4 oriente & Arquitectura pobre, cancro y muerte regresiva de copa \\
\hline 14 & Acer negundo & 1 norte con 4 oriente & $\begin{array}{l}\text { Madera descompuesta, cavidad, unión débil de rama y } \\
\text { cancro }\end{array}$ \\
\hline 15 & Platanus orientalis & 5 norte con 4 oriente & Arquitectura pobre, inclinación y grietas \\
\hline 16 & Platanus orientalis & 5 norte con 4 oriente & Arquitectura pobre y grietas \\
\hline 17 & Platanus orientalis & 9 norte con 5 oriente & Arquitectura pobre y cavidad \\
\hline 18 & Melia azadarach & 5 sur con 4 oriente & Inclinación y grietas \\
\hline 19 & Eucalyptus sp. & 3 sur con $111 / 2$ poniente & Grieta, muerte regresiva de copa y ramas muertas \\
\hline 20 & Acer negundo & 4 norte con 4 oriente & Arquitectura pobre y cancros \\
\hline 21 & Platanus orientalis & 5 norte con 6 oriente & Arquitectura pobre \\
\hline 22 & Platanus orientalis & 5 oriente con 3 norte & Arquitectura pobre \\
\hline 23 & Araucaria angustifolia & $161 / 2$ sur con 1 oriente & Inclinación \\
\hline 24 & Catalpa speciosa & 15 sur con Pasaje Tirso de Molina & Madera descompuesta y grieta \\
\hline 25 & Acer negundo & 14 sur con Pasaje Tirso de Molina & Arquitectura pobre, grietas y cancros \\
\hline 26 & Robinia pseudoacacia & 9 sur con Pasaje Fresia & Grieta e inclinación \\
\hline 27 & Acer negundo & 9 sur con Pasaje Fresia & Grieta, cavidad, inclinación y muerte regresiva de copa \\
\hline 28 & Morus nigra & 9 sur con Pasaje Fresia & Inclinación \\
\hline 29 & Casuarina equisetifolia & 7 sur con 1 oriente & Madera descompuesta y arquitectura pobre \\
\hline 30 & Prunus cerasifera & 3 sur con 2 poniente & Madera descompuesta, inclinación, grieta y cavidad \\
\hline
\end{tabular}


Las metodologías de evaluación visual del riesgo en el arbolado urbano usadas se resumen a continuación:

A) Avaliação de árvores de risco na arborização de vias públicas de Nova Olímpia, Paraná (Método Sampaio [Sampaio, Duarte, Silva, De Angelis y Blum, 2010]), desarrollado para el arbolado urbano en el municipio de Nova Olimpia, Estado de Paraná, Brasil. Se compone de dos partes: la primera consiste en la evaluación general de las condiciones del árbol, que subdivide en tres las zonas de evaluación: dosel, tronco y base del tronco; cada una compuesta por distintos indicadores relacionados con el estado fitosanitario, condiciones del lugar, estructura y tamaño del árbol. Los indicadores se califican en función de una determinada característica que indica riesgo, donde 0 significa que esta característica no está presente en el árbol y de 1 a 5 significa un aumento de esta característica. La segunda parte consta de dos ítems de puntuación, el primero es el índice de riesgo para redes eléctricas, relacionado con la cercanía del árbol al tendido eléctrico, puntuación que varía entre 1, 3 y 5 puntos; el segundo es el índice de riesgo para efecto colateral, vinculado con la probabilidad de daño a las personas, en el que se evalúa la zona y su grado de ocupación, su puntuación varía entre 1, 3 y 5 . El índice de riesgo final, que varía de 3 a 15 puntos, se compone del mayor grado de riesgo obtenido en la evaluación de las condiciones generales del árbol, sumado al índice de riesgo para redes eléctricas y el índice de riesgo para efecto colateral. Un índice de 3 a 6 representa un riesgo menor; un índice de 7 a 11 tiene un riesgo de media importancia; y el índice de 12 a 15 corresponde a un riesgo de elevada importancia.

B) Urban Tree Risk Management: A Community Guide to Program Design and Implementation (Pokorny, 2003 [Método USF]), permite la identificación de árboles defectuosos al evaluar las consecuencias de fallas y entrega recomendaciones de acciones destinadas a reducir su riesgo (Koeser, Hasing, McLean y Northrop, s.f.). Se aplica en la medida que exista un bien, persona o servicio que pueda ser potencialmente dañado, denominado blanco, el cual debe encontrarse a una distancia menor a 1.5 veces la altura del árbol (Pokorny, 2003). La metodología es un sistema de calificación de 10 puntos, compuestos por cuatro secciones de análisis: probabilidad de fallo, tamaño de la pieza defectuosa, probabilidad de impacto y otros riesgos, como calificación opcional. La probabilidad de fallo evalúa la presencia de defectos que debiliten y deterioren la estructura del árbol, la calificación de riesgo se divide en: baja (1), moderada (2), alta (3), y extrema (4). La sección otros riesgos considera asignar de uno o dos puntos opcionales, si a juicio del evaluador existe la necesidad de aumentar la calificación de riesgo, a fin de ejecutar acciones correctivas inmediatas; la valoración total del riesgo no debe superar los 10 puntos. La calificación final de riesgo es la suma de los tres componentes principales y, opcionalmente, el cuarto componente. Los árboles con la calificación de riesgo numérico más alto (10) deben ser tratados en primer lugar, con base en la política del municipio, eliminar o tratar los árboles defectuosos con los índices más altos e ir bajando hasta que lo permitan los recursos financieros y humanos (Pokorny, 2003).

C) A Photographic Guide to the Evaluation of Hazard Trees in Urban Areas (Matheny y Clark, 1994; Método ISA[A]), desarrollado bajo la International Society of Arboriculture y ampliamente utilizado y modificado por municipios y arboristas comerciales (Koeser, Hasing, McLean y Northrop, s.f.; Calaza e Iglesias, 2016). Tiene un procedimiento cuyo protocolo de análisis está dividido en cinco secciones: características del árbol, estado fitosanitario, condición de sitio, blanco y defectos del árbol. En cada una de estas secciones se determina la gravedad de los defectos individuales y se identifica la o las partes del árbol con mayor probabilidad de fallo. La información recopilada permite calificar el riesgo del árbol mediante tres índices de puntuación: potencial falla (1-3 puntos), tamaño de la parte (1-4 puntos), puntaje de uso (1-4 puntos). Los puntajes alcanzados en cada sección 
se suman para obtener el índice final de riesgo, siendo 12 el máximo valor. Las puntuaciones tienen solo un significado relativo, un árbol puntuado con 11 tiene un mayor riesgo potencial que uno con 5, por lo que un árbol puntuado con 12 significa un peligro extremo, aunque la eliminación de este peligro puede ser tan simple como mover el blanco bajo riesgo (Calaza e Iglesias, 2016).

D) Best Management Practice, Tree Risk Assessment (Smiley, Matheny y Lilly, 2011) (Método $[S A[B])$, es un método diseñado para árboles que reciben la clasificación nivel dos dentro de los tres niveles de evaluación de riesgo definidos en la norma ANSI A300 (Standards for Tree Care Operations) (ANSI, 2011). Recoge la mayoría de los aspectos analizados por el método ISA(A), pero califica el riesgo en categorías descriptivas, ya que los usuarios son guiados a través de matrices de decisión para determinar la calificación de riesgo general. El protocolo de análisis se encuentra a modo de lista de chequeo y está dividido en cinco secciones: evaluación del blanco, factores de sitio, salud del árbol y perfil de la especie, factores de carga y defectos que inciden en la probabilidad de falla. Se analiza la o las partes del árbol que pueden fallar, evaluando el tamaño de la parte, la distancia de caída hacia el blanco y el blanco. Luego se determina la probabilidad de falla de la parte y la probabilidad de impacto del blanco, para posteriormente determinar las consecuencias de la falla. La clasificación final del riesgo resulta de la probabilidad de falla e impacto combinada con las consecuencias de la falla, los resultados de ambas se introducen en una matriz de clasificación de riesgo la que genera la calificación final del riesgo de la parte o del árbol completo, que se categoriza en: bajo, moderado, alto o extremo (tabla 2).

Las evaluaciones fueron realizadas entre el 19 de diciembre del 2014 y el 22 de enero del 2015. Se midieron las variables dendrométricas de altura, diámetro a la altura del pecho y diámetro de copa. Para la evaluación visual del riesgo se utilizaron

Tabla 2. Características de los métodos

\begin{tabular}{|c|c|c|c|c|}
\hline \multirow[b]{2}{*}{ Características } & \multicolumn{4}{|c|}{ Métodos } \\
\hline & Sampaio & USF & $\operatorname{ISA}(\mathrm{A})$ & ISA $(B)$ \\
\hline $\begin{array}{l}\text { Factores de riesgo } \\
\text { calificados }\end{array}$ & $\begin{array}{l}\text { Condición general del } \\
\text { árbol } \\
\text { Uso del espacio } \\
\text { Cercanía a redes eléc- } \\
\text { tricas }\end{array}$ & $\begin{array}{l}\text { Probabilidad de falla } \\
\text { Tamaño de la pieza } \\
\text { Uso del espacio }\end{array}$ & $\begin{array}{l}\text { Probabilidad de falla } \\
\text { Tamaño de la pieza } \\
\text { Uso del espacio }\end{array}$ & $\begin{array}{l}\text { Probabilidad de falla } \\
\text { Uso del espacio } \\
\text { Consecuencias de la } \\
\text { falla }\end{array}$ \\
\hline Tipo de calificación & Numérica & Numérica & Numérica & Descriptiva \\
\hline $\begin{array}{l}\text { Rango de calificación } \\
\text { de riesgo }\end{array}$ & De 3 a 15 & De 3 a 10 & De 3 a 12 & $\begin{array}{l}\text { Bajo } \\
\text { Moderado } \\
\text { Alto } \\
\text { Extremo }\end{array}$ \\
\hline $\begin{array}{l}\text { Tiempo de análisis } \\
\text { aproximado (minutos) }\end{array}$ & 15 a 20 & 10 a 15 & 20 a 25 & 20 a 25 \\
\hline Particularidades & $\begin{array}{l}\text { En la sección análisis } \\
\text { general del árbol, todos } \\
\text { los indicadores reciben } \\
\text { puntaje, pero solo se } \\
\text { considera el mayor } \\
\text { valor alcanzado en } \\
\text { cualquiera de las tres } \\
\text { zonas de análisis (copa, } \\
\text { tronco y base) }\end{array}$ & $\begin{array}{l}\text { Considera un puntaje } \\
\text { opcional en el caso } \\
\text { que exista algún factor } \\
\text { no contemplado en la } \\
\text { evaluación o si a juicio } \\
\text { del evaluador amerite } \\
\text { aumentar la calificación } \\
\text { de riesgo }\end{array}$ & $\begin{array}{l}\text { El formulario de evalua- } \\
\text { ción contiene los facto- } \\
\text { res de riesgo a partir de } \\
\text { los cuales se determina } \\
\text { la probabilidad de falla } \\
\text { del árbol }\end{array}$ & $\begin{array}{l}\text { La calificación de final } \\
\text { riesgo se realiza me- } \\
\text { diante dos matrices de } \\
\text { análisis }\end{array}$ \\
\hline
\end{tabular}


formularios de los métodos para la toma de datos, de acuerdo a cada metodología en particular. Los índices de riesgo obtenidos de las evaluaciones se procesaron en una planilla de cálculo de Excel®.

Dada la particularidad de cada método en relación al índice final de riesgo, los resultados obtenidos se estandarizaron, de modo que la variación de la puntuación del riesgo para cada método se expresó en un rango de 0 a 100\%, haciendo uso de la siguiente expresión:

$$
\text { IE }=(\text { I - val mín. }) *(100 / \text { (val máx. - val mín. }))
$$

Donde, IE: índice de riesgo estandarizado; I: índice de riesgo; val mín.: valor mínimo a alcanzar por el método; val máx.: valor máximo a alcanzar por el método.

Para realizar la comparación se establecieron cuatro niveles de riesgo: riesgo bajo (RB) de 0 a $25 \%$; riesgo medio (RM) mayor a 25 y hasta $50 \%$; riesgo alto (RA) mayor a 50 y hasta $75 \%$; y riesgo extremo (RE) mayor a $75 \%$.

En el análisis estadístico se planteó la siguiente hipótesis:

$H_{0}: \beta_{1}=\beta_{2} \ldots=\beta_{k^{\prime}}$ no existen diferencias estadísticamente significativas entre las medias de los índices de riesgo de los métodos evaluados.

$\mathrm{H}_{1}: \beta_{1} \neq \beta_{2} \ldots \neq \beta_{\mathrm{k}^{\prime}}$ existen diferencias estadísticamente significativas entre las medias de los índices de riesgo de los métodos evaluados.

Donde $\beta_{1 \ldots} \beta_{k^{\prime}}$ representan las medias.

Se aplicó el test de Levene para el análisis de homogeneidad de varianza y la prueba de Shapiro-Wilk para determinar si los datos provienen de una distribución normal, obteniéndose valores de $p=0.0248927$ y $p=0.000161588(p<0.05)$ respectivamente, condición que se mantuvo incluso realizándose técnicas de normalización de los datos. Los resultados determinaron que no se cumplen los supuestos subyacentes al análisis de varianza, por lo cual se procedió a realizar el análisis mediante estadística no paramétrica. Se utilizó el análisis de varianza de Kruskal-Wallis, que no requiere suposiciones de normalidad de los datos (WackerIy, Mendehall y Scheaffer, 2010), obteniéndose un valor $P=9.31286^{*} 10^{-10}(\mathrm{p}<0.05)$, lo que indicó la existencia de diferencias significativas entre los métodos.

La hipótesis para los métodos se replanteó de la siguiente manera:

$H_{0}: \Omega_{1}=\Omega_{2} \ldots=\Omega_{\mathrm{k}^{\prime}}$ no existen diferencias estadísticamente significativas entre las medianas de los índices de riesgo de los métodos evaluados.

$H_{1}: \Omega_{1} \neq \Omega_{2} \ldots \# \Omega_{\mathrm{k}^{\prime}}$ existen diferencias estadísticamente significativas entre las medianas de los índices de riesgo de los métodos evaluados.

Donde $\Omega_{1} \ldots \Omega_{\mathrm{k}}$ representan las medianas.

Para determinar la existencia de una o más fuentes de variación se utilizó el test de rangos múltiples de Duncan, concordante otros estudios (Hernández, Arreaza y Lazo, 2002; Cárdenas, 2008; Merchán-Gaitán, Ferrucho y Álvarez-Herrera, 2014).

Los materiales utilizados fueron: formularios, forcípula, huincha métrica, clinómetro, transportador, planilla MS-Excel 2013 y el software Statgraphcis Centurion XVI®.

\section{RESULTADOS}

Se obtuvo los índices de riesgo para cada árbol evaluado y su índice estandarizado para cada uno de los métodos (tabla 3).

Los principales estadígrafos muestran diferencias entre los métodos al calificar el riesgo en un mismo árbol (tabla 4). En cuanto al índice estandarizado, el promedio de los métodos ISA(A) y USF se ubican cercano a 50.0, mientras que en los extremos están el método ISA(B) en la parte baja (29.2) y el método Sampaio en la parte alta (61.1); misma tendencia se observa tanto en la mediana como en la moda. La desviación estándar es menor en los métodos ISA, siendo más aun en el método $\operatorname{ISA}(\mathrm{B})$. 
Tabla 3. Índice de riesgo y valor estandarizado para cada árbol según método

\begin{tabular}{ccccccccc}
\hline \multirow{2}{*}{$\mathbf{N}^{\circ}$ Árbol } & \multicolumn{2}{c}{ Método Sampaio } & \multicolumn{2}{c}{ Método USF } & \multicolumn{2}{c}{ Método ISA(A) } & \multicolumn{2}{c}{ Método ISA(B) } \\
\cline { 2 - 8 } & Índice & I E* $^{*}$ & Índice & IE* $^{*}$ & Índice & I E* & Índice & I E* $^{*}$ \\
\hline 1 & 13 & 83.3 & 4 & 14.3 & 4 & 11.1 & Bajo & 12.5 \\
2 & 7 & 33.3 & 6 & 42.9 & 7 & 44.4 & Bajo & 12.5 \\
3 & 7 & 33.3 & 6 & 42.9 & 7 & 44.4 & Bajo & 12.5 \\
4 & 13 & 83.3 & 5 & 28.6 & 5 & 22.2 & Bajo & 12.5 \\
5 & 13 & 83.3 & 7 & 57.1 & 8 & 55.6 & Moderado & 37.5 \\
6 & 13 & 83.3 & 7 & 57.1 & 8 & 55.6 & Moderado & 37.5 \\
7 & 9 & 50.0 & 8 & 71.4 & 8 & 55.6 & Moderado & 37.5 \\
8 & 13 & 83.3 & 6 & 57.1 & 8 & 55.6 & Moderado & 37.5 \\
9 & 9 & 50.0 & 6 & 57.1 & 7 & 44.4 & Moderado & 37.5 \\
10 & 13 & 83.3 & 8 & 71.4 & 9 & 66.7 & Alto & 62.5 \\
11 & 13 & 83.3 & 8 & 71.4 & 8 & 55.6 & Bajo & 12.5 \\
12 & 11 & 66.7 & 4 & 14.3 & 5 & 22.2 & Bajo & 12.5 \\
13 & 11 & 66.7 & 4 & 42.9 & 5 & 22.2 & Bajo & 12.5 \\
14 & 11 & 66.7 & 9 & 85.7 & 9 & 66.7 & Moderado & 37.5 \\
15 & 13 & 83.3 & 7 & 57.1 & 8 & 55.6 & Moderado & 37.5 \\
16 & 13 & 83.3 & 7 & 57.1 & 8 & 55.6 & Moderado & 37.5 \\
17 & 13 & 83.3 & 7 & 57.1 & 8 & 55.6 & Moderado & 37.5 \\
18 & 13 & 83.3 & 6 & 42.9 & 6 & 33.3 & Bajo & 12.5 \\
19 & 6 & 25.0 & 5 & 28.6 & 6 & 33.3 & Bajo & 12.5 \\
20 & 15 & 1000 & 7 & 57.1 & 8 & 55.6 & Moderado & 37.5 \\
21 & 9 & 50.0 & 6 & 42.9 & 7 & 44.4 & Moderado & 37.5 \\
22 & 9 & 50.0 & 6 & 42.9 & 6 & 33.3 & Moderado & 37.5 \\
23 & 9 & 50.0 & 9 & 87.5 & 10 & 77.8 & Alto & 62.5 \\
24 & 9 & 50.0 & 8 & 71.4 & 10 & 77.8 & Moderado & 37.5 \\
25 & 6 & 25.0 & 4 & 14.3 & 9 & 66.7 & Bajo & 12.5 \\
26 & 7 & 33.3 & 5 & 28.6 & 5 & 22.2 & Bajo & 12.5 \\
27 & 7 & 33.3 & 5 & 28.6 & 5 & 22.2 & Bajo & 12.5 \\
28 & 6 & 25.0 & 5 & 28.6 & 6 & 33.3 & Bajo & 12.5 \\
29 & 10 & 58.3 & 8 & 71.4 & 9 & 66.7 & Moderado & 37.5 \\
30 & 9 & 50.0 & 8 & 71.4 & 8 & 55.6 & Alto & 62.5 \\
\hline
\end{tabular}

*: índice estandarizado

Tabla 4. Principales estadígrafos

\begin{tabular}{lcccccccc}
\hline \multirow{2}{*}{ Estadígrafo } & \multicolumn{2}{c}{ Método ISA(B) } & \multicolumn{2}{c}{ Método ISA(A) } & \multicolumn{2}{c}{ Método Sampaio } & \multicolumn{2}{c}{ Método USF } \\
\cline { 2 - 8 } & Índice & $\begin{array}{c}\text { Índice } \\
\text { estandarizado }\end{array}$ & Índice & $\begin{array}{c}\text { Índice } \\
\text { estandarizado }\end{array}$ & Índice & $\begin{array}{c}\text { Índice } \\
\text { estandarizado }\end{array}$ & Índice & $\begin{array}{c}\text { Índice } \\
\text { estandarizado }\end{array}$ \\
\hline Promedio & --- & 29.2 & 7.2 & 47.0 & 10.3 & 61.1 & 6.4 & 48.6 \\
Mediana & --- & 37.5 & 8.0 & 55.6 & 10.5 & 62.5 & 6.5 & 50.0 \\
Desv. St. & --- & 16.5 & 1.6 & 17.9 & 2.7 & 22.7 & 1.5 & 21.5 \\
Desv. St. (\%) & --- & 56.5 & 22.2 & 38.1 & 26.2 & 37.2 & 23.4 & 44.2 \\
Moda & --- & 37.5 & 8.0 & 55.6 & 13.0 & 83.3 & 7.0 & 57.1 \\
\hline
\end{tabular}


El método que obtuvo más evaluaciones de árboles en riesgo extremo fue Sampaio (12), con riesgo alto fue el método ISA(A) con 14 evaluaciones, seguido de USF con 13. El mayor número de árboles evaluados con riesgo moderado (14) fue con el método ISA(B), seguido de los métodos Sampaio y USF (11). El método que evaluó más árboles en riesgo bajo fue $\mathrm{ISA}(\mathrm{B})$ con 13 , seguido del ISA(A) con 7 (figura 1).

La aplicación del test de Kruskal-Wallis identificó diferencias estadísticas de las evaluaciones y el test de Duncan cómo se agrupan los métodos (figura 2, figura 3), en el que se identificó que los cuatro métodos se agrupan en tres grupos. Además, con

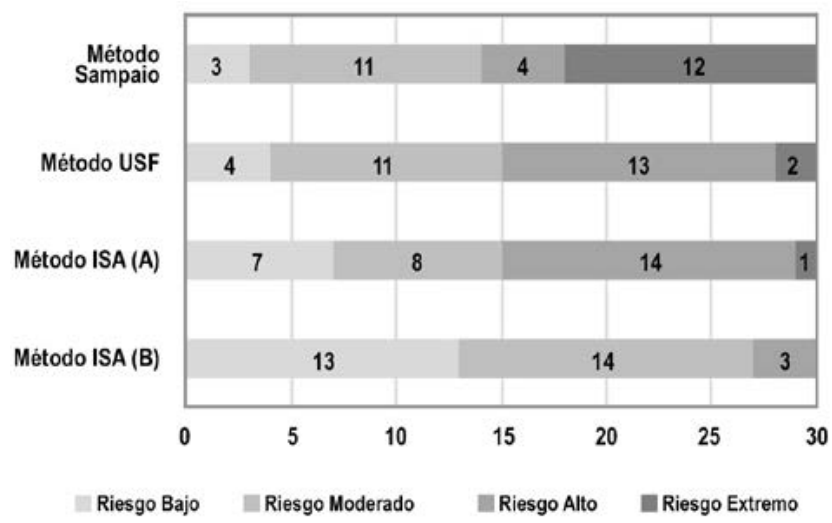

Figura 1. Número de árboles según clasificación de riesgo.

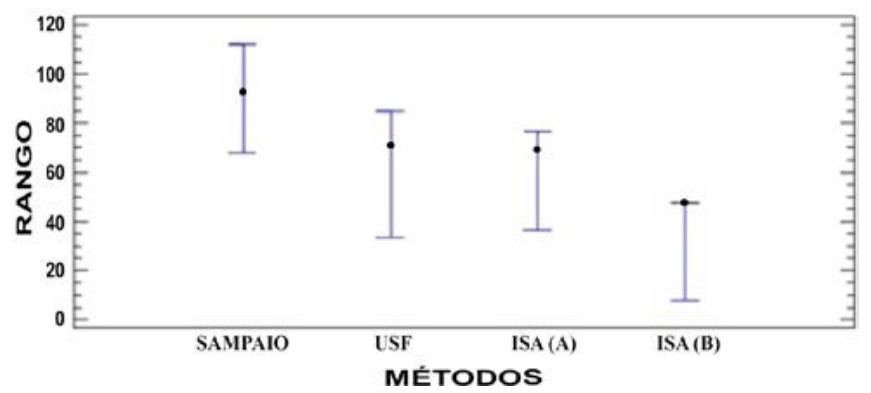

Figura 2. Gráfico de medianas con intervalos del 95\% de confianza.

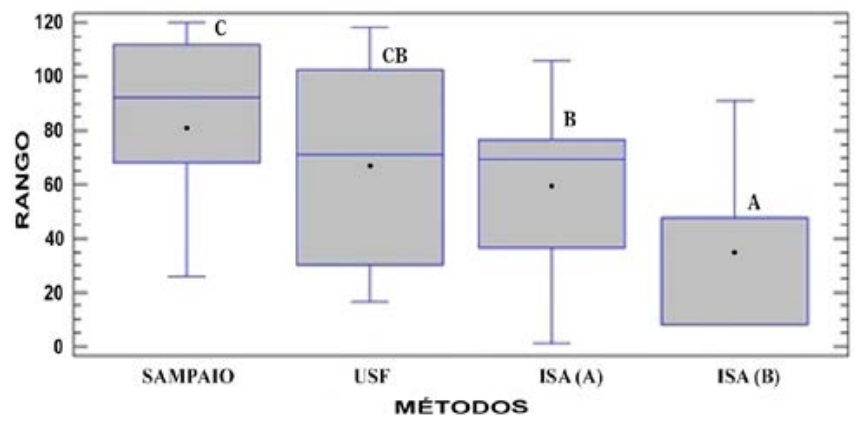

Figura 3. Gráfico de caja y bigote para rangos por métodos. Las letras sobre las cajas muestran los tres grupos conformados al aplicar el test de Duncan (A, B y C). 
la finalidad de identificar mejor su variabilidad se obtuvo el gráfico de caja y bigote (figura 3), en el cual se observó la variabilidad que muestran los desempeños de cada uno de los métodos.

\section{DISCUSIÓN}

Los métodos de evaluación visual básica usados en este trabajo han resultado ser un medio eficiente para determinar el riesgo del árbol urbano, dado a que junto con la factibilidad de aplicación logran identificar niveles de riesgo del árbol evaluado, por lo que son métodos viables, tal como lo señala Koeser, Hauer, Klein y Miesbauer (2017), respecto de otras metodologías como las de limitación visual o instrumental. Aun cuando presentaron un desempeño diferenciado.

En general, las diferencias encontradas se deben a la estructura de los métodos, ya que la multidimensionalidad que el riesgo representa en la evaluación se ve expresada en las diferentes variables y su peso relativo en cada uno de los métodos (Norris, 2007; Tomao et al., 2015; Koeser y Smiley, 2017). Así, el método Sampaio hace énfasis en la cercanía a redes del tendido eléctrico aéreo, considerando que, según datos de 2010 de la Agencia Nacional de Energía Eléctrica de Brasil (ANEEL), la utilización de redes subterráneas de distribución no llegaba a los $2 \%$ del total de redes de baja y media tensión en ese país (Nakaguishi y Hermes, 2011). Por lo tanto, la aplicación del método Sampaio sería recomendable donde la mayoría del cableado de distribución de energía sea subterráneo, situación que no sucede en la ciudad de Talca, lugar del estudio.

Comparativamente, el método que muestra un desempeño diferenciado de los desarrollados en ISA, en este estudio es el método ISA(A). Por su parte, ISA(B) identificó riesgo solo en tres de las cuatro categorías establecidas; al tiempo que ISA(A) lo hizo en las cuatro, con una mejor distribución, calificó 7 árboles en riesgo bajo, 8 en riesgo medio, 14 en riesgo alto y solo uno en riesgo extremo (figura 1); e ISA(B) concentró sus resultados en riesgo bajo (13) y riesgo moderado (14).

En comparación al estudio de Norris (2007), tanto el promedio como la desviación estándar de los métodos ISA(A) y USF, incorporados en ambos estudios, fueron similares. ISA(A) mostró en este estudio una diferencia en el promedio de $0.73(10.1 \%)$ y para USF la diferencia fue de -0.13 (-2\%); de otra parte, en la desviación estándar, las diferencias fueron de $0.3(2.2 \%)$ para ISA(A) y de $0.1(1.4 \%)$ en USF. Lo anterior permite señalar que ambos métodos mostraron similares desempeños en ambos estudios y, como se observó, conformaron uno de los grupos del análisis de rangos.

El test de rangos múltiples de Duncan identificó tres grupos homogéneos para los cuatro métodos (figura 3). El método ISA(B) conforma el grupo A, que presentó las evaluaciones de riesgo más bajos en el ranking; los métodos ISA(A) y USF conforman el grupo $B$, con valores de riesgo en rangos intermedios, relación que también pudo observarse en la comparación del estudio hecho por Norris (2007); mientras que USF y método Sampaio constituyen el grupo C, con los valores de riesgo más altos. Estos resultados se observan con mayor claridad en el gráfico de medianas con intervalos, con 95\% de confianza (figura 2). En esta figura se aprecia que la mediana del método Sampaio presentó un valor centrado en el rango, para el caso de los métodos USF e ISA(A) se ubicó en valores más altos y para el método ISA(B) se posicionó en el extremo de los valores altos dentro del ranking.

El gráfico de caja y bigote (figura 3) mostró que las evaluaciones de riesgo realizadas con el método USF obtuvieron una mejor distribución de las evaluaciones, mostrando una amplia dispersión, cuya media se ubicó muy cercana a la mediana. Le siguió en desempeño el método Sampaio que, aunque con evaluaciones de riesgo con niveles relativos más altos, la mediana se ubicó en el centro del cuartil inferior. A su vez, el método ISA(A) concentra gran parte de las evaluaciones en el cuartil superior y la media se ubicó al interior del cuartil inferior, siendo el principal aspecto positivo la 
mayor dispersión de datos en todos los métodos. Lo anterior mostraría una mejor capacidad de discriminar los diferentes niveles de riesgo, aspecto además relacionado con la amplitud de la puntuación del método. El método ISA(B) presentó en esta gráfica (figura 3) un desempeño con limitaciones, dado que la transformación de las cuatro expresiones cualitativas del método original a cuatro cuantitativas, hace que el análisis cuantitativo resulte con serias restricciones, ya que los datos se concentraron en un único cuartil, esto es en la parte baja de las calificaciones de riesgo.

Finalmente, dado el contexto de esta investigación, sus resultados y las investigaciones referenciadas, se sugiere ampliar este tipo de estudios en los que se incorpore un número mayor de árboles y de evaluadores, de tal manera que ampliando la base de las fuentes de variación se logre mayor certidumbre respecto al método a usar.

\section{CONCLUSIONES}

Las metodologías de evaluación visual del riesgo para el arbolado urbano constituyen una herramienta eficiente, ya que se basan en criterios técnicos, biológicos y biomecánicos apoyados en la experticia necesaria del evaluador. Sin embargo, no todas las usadas en este estudio serían recomendadas para los espacios urbanos arbolados de Chile.

Los resultados evidenciaron diferencias estadísticas significativas en los métodos evaluados, conformando tres grupos homogéneos. El método Sampaio conformó un grupo, obteniendo índices de riesgo más altos, mientras un segundo grupo lo conformaron los métodos USF e ISA(A), con índices de riesgo medios a altos, y el tercer grupo fue constituido por el método ISA(B) que obtuvo valores bajos en la calificación de riesgo.

De acuerdo a los resultados, no sería recomendable aplicar el método Sampaio en zonas donde habitualmente hay redes eléctricas, condición habitual en las ciudades de Chile central, ya que es un factor que sobreestima la calificación de riesgo, obteniéndose valores de riesgo muy altos.

Se recomienda la aplicación de los métodos ISA(A) y USF, teniendo en cuenta los resultados obtenidos y su análisis estadístico y en consideración a las condiciones generales donde se desarrollan los árboles en zonas urbanas de Chile, dado que sería más inequívoca la determinación del riesgo del árbol.

Es necesario seguir investigando, validando y ajustando métodos para las diferentes condiciones locales y contar con más evaluadores para reducir la incertidumbre de las evaluaciones.

\section{CONFLICTO DE INTERESES}

Los autores declaran no tener conflicto de intereses.

\section{CONTRIBUCIÓN POR AUTOR}

Los autores son los únicos responsables de la obra en todos los aspectos que condujeron a la elaboración de su publicación.

\section{REFERENCIAS BIBLIOGRÁFICAS}

American National Standard Institute (ANSI) (2011). Tree, Shrub, and Other Woody Plant Management - Standard Practices. Ansi A300 (Part 9) - Tree Risk Assessment. Washington, DC: American National Standards Institute.

Angwin, P., Cluck, D., Zambino, P., Oblinger, B. y Woodruff, W. (2012). Hazard Tree Guidelines for Forest Service Facilities and Road in the Pacific Southwest Region. Recuperado de http://www.fs.usda.gov/lnternet/FSE_DOCUMENTS/stelprdb5332560.pdf

Biblioteca del Congreso Nacional (BCN) (2017). Reportes estadísticos distritales y comunales 2013. Recuperado de http://reportescomunales.bcn.cl

Bonells, J. (2003). La gestión moderna del arbolado urbano de las ciudades. Sevilla, España. Recuperado 
de http://www.sevilla.org/ayuntamiento/areas/ area-de-urbanismo-y-medio-ambiente/aservicio-de-parques-y-jardines/e-articulos-tecnicos/.pdf

Calaza, P. e Iglesias, M. (2016). El riesgo del arbolado urbano. Contexto, concepto y evaluación. Madrid: Editorial Mundiprensa.

Cárdenas, C. (2008). Identificación de tipologías de actitud hacia las matemáticas en estudiantes de séptimo y octavo grados de educación primaria. Perfiles educativos, 30(122), 94-108.

Ellison, M. (2005). Quantified Tree Risk Assessment used in the management of amenity trees. Journal of Arboriculture, 31(2), 57-65.

Food and Agricultural Organization (FAO) (2017). Directrices para la silvicultura urbana. Estudio FAO: Montes 178. Roma: FAO.

Forrest, M. (2002). Trees in European cities - a historical review. En L. Dunne (ed.), Biodiversity in the city (pp. 15-22). Dublín: University Collage Dublín, Environmental Institute.

Geiger, J., King, C. y Hartel, D. (2004). The Large Tree Argument - The case for large-stature trees vs. small-stature trees. Davis, CA: Center for Urban Forest Research, Pacific Southwest Research Station, USDA Forest Service. Recuperado de https:// www.fs.fed.us/psw/topics/urban_forestry/products/ cufr_511_large_tree_argument.pdf

Hauer, R. y Johnson, G. (2003). Tree Risk Management in Urban tree risk management: A community guide to program design and implementation. Saint Paul, EE. UU.: USDA Forest Service. Recuperado de http://www.na.fs.fed.us/spfo/pubs/uf/utrmm/urban_tree_risk_mgmnt.pdf

Hernández, L., Arreaza, J. y Lazo, A. (2002). Evaluación de nicosulfuron en el control de Rottboellia exaltata, Euphorbia heterophylla y Aldama dentata en el cultivo de maíz (Zea mays L.) aplicado en tres diferentes estadios de desarrollo de las malezas. Revista de la Facultad de Agronomía 19(4), 294-303.

James, K.R. (2010). A dynamic structural analysis of trees subject to wind loading (tesis de doctorado). Melbourne School of Land and Environments, The University of Melbourne, Melbourne, Australia.
Koeser, A. y Smiley, T. (2017). Impact of assessor on tree risk assessment rating and prescribed mitigation measures. Urban Forestry \& Urban Greening, 24, 109-115. DOI: https://doi.org/10.1016/j. ufug.2017.03.027

Koeser, A., Hasing, G., McLean, D. y Northrop, R. (s.f.). Tree risk assessment methods: A comparison of three common evaluation forms (ENH1226). Florida, EE. UU.: University of Florida, Institute of Food and Agricultural Sciences. Recuperado de http:// www.edis.ifas.ufl.edu/ep487

Koeser, A., Hauer, R.J., Klein, R. W. y Miesbauer, J.W. (2017). Assessment of likelihood of failure using limited visual, basic, and advanced assessment techniques. Urban Forestry \& Urban Greening, 24, 71-79. DOI: https://doi.org/10.1016/j. ufug.2017.03.024

Koeser, A., Hauer, R., Miesbauer, J. y Peterson, W. (2016). Municipal tree risk in the United States: Findings from a comprehensive survey of urban forest management. Arboriculture Journal, 38(4), 218229. DOI: https://doi.org/10.1080/03071375.201 6.1221178

Konijnendijk, C., Kjell, N., Randrup, T. y Schipperijn, L. (2005). Urban Forest and Trees. Amsterdam, Holanda: Springer Verlag. DOI: https://doi. org/10.1007/3-540-27684-X

Matheny, N. y Clark, J. (1994). A photographic guide to the evaluation of hazard trees in urban areas (2 ${ }^{\mathrm{a}}$. ed.). Savoy, IL: International Society of Arboriculture.

Mattheck, C. y Breloer, H. (1994). The body language of trees: a handbook for failure analysis. Londres: HMSO Publications Centre. DOI: https://doi. org/10.1007/BF00197869

Merchán-Gaitán, J., Ferrucho, R. y Álvarez-Herrera, J. (2014). Efecto de dos cepas de Trichoderma en el control de Botrytis cinereay la calidad del fruto en fresa (Fragaria sp.). Revista Colombiana de Ciencias Hortícolas, 8(1), 44-56

Miller, R., Hauer, R. y Werner, L. (2015). Urban Forestry. Planning and Managing Urban Greenspaces ( $3^{\mathrm{a}}$ ed.). Illinois: Waveland Press, Inc. DOI: https://doi. org/10.1016/j.ufug.2015.09.001 
Nakaguishi, M. y Hermes, P. (2011). Estudo comparativo técnico/financeiro para implantação de redes de distribuição subterrâneas (tesis de grado). Universidade Federal do Paraná, Curitiba, Brasil.

National Tree Safety Group (NTSG) (2011). Common sense risk management of trees: Guidance on trees and public safety in the UK for owners, managers and advisers. Edimburgo: Forestry Commission. Recuperado de http://www.forestry.gov.uk/pdf/ FCMS024.pdf

Norris, M. (2007). Tree risk assessment - What works What does not - Can we tell? A review of a range of existing tree risk assessment methods. Ponencia presentada en el ISAAC Conference Perth 2007.

O'Brien, J. (2003). Introduction. En, Urban Tree Risk Management: A Community Guide to Program Design and Implementation. Saint Paul, EE. UU.: USDA Forest Service. Recuperado de http://www. na.fs.fed.us/spfo/pubs/uf/ utrmm/urban_tree_risk _ mgmnt.pdf. https://doi.org/10.1201/b16997-2

Pauleit, S., Jones, N., García-Marín, G., García-Valdecantos, J.L., Riviẻre, L.M., Vidal-Beaudet, L., Bodson, M. y Randrup, T. (2002). Tree stablishment practice in towns and cities - results from a European survey. Urban Forestry \& Urban Greening, 1(2), 8396. DOI: https://doi.org/10.1078/1618-8667-00009

Pokorny, J. (2003). Urban tree risk management: A community guide to program design and implementation. Saint Paul, EE. UU.: USDA Forest Service. Recuperado de http://www.na.fs.fed.us/spfo/pubs/ uf/utrmm/urban_tree_risk_mgmnt.pdf

Ponce-Donoso, M. y Vallejos-Barra, O. (2016). Valoración de árboles urbanos, comparación de fórmulas. Revista de la Facultad de Ciencias Agrarias de la Universidad Nacional de Cuyo, 48(2), 195-208.
Restrepo, H. I., Moreno, F. y Hoyos, C. (2015). Incidencia del deterioro progresivo del arbolado urbano en el Valle de Aburrá, Colombia. Colombia Forestal, 18(2), 225-240. DOI: https://doi.org/10.14483/ udistrital.jour.colomb.for.2015.2.a04

Sampaio, A., Duarte, F., Silva, E., De Angelis, B. y Blum, C. (2010). Avaliação de árvores de risco na arborização de vías públicas de Nova Olímpia, Paraná. Revista da Sociedade Brasileira de Arborização Urbana, 5(2), 82-104.

Smiley, E., Matheny, N. y Lilly, S. (2011). Best Management Practices: Tree Risk Assessment. Champaign, IL: International Society of Arboriculture.

Tomao, A., Secondi, A., Corona, P., Giuliarelli, D., Quantrini, V. y Agrimi, M. (2015). Can composite indices explain multidimensionality of tree risk assessment? A case study in an historical monumental complex. Urban Forest \& Urban Greening, 14(3), 456-465. DOI: https://doi.org/10.1016/j. ufug.2015.04.009

Tyrväinen, L., Silvennoinen, H. y Kolehmainen, O. (2003). Ecological and aesthetic value in urban forest management. Urban Forest \& Urban Greening, 1(3), 135-149. DOI: https://doi. org/10.1078/1618-8667-00014

Van Wassenaer, P. y Richardson, M. (2009). A Review of Tree Risk Assessment Using Minimally Invasive Technologies and Two Case Studies. Arboricultural Journal, 32, 275-292. DOI: https://doi.org/10.1080 103071375.2009.9747583

Wackerly, D., Mendehall, W. y Scheaffer, R. (2010). Estadística matemática con apliaciones ( $7^{\mathrm{a}}$ ed.). México D.F.: Cengage Learning Editores.

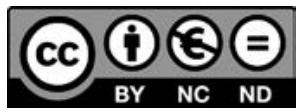

